Introduction Sleepiness during work hours is the most common complaint of night shift workers, and is a sensitive indicator of performance decrements. Sleepiness levels vary between individuals, yet few have investigated individual factors as predictors of night shift sleepiness, and these have shown mixed results. We aimed to examine the effects of biopsycho-social factors on subjective sleepiness of nurses during the night shift.

Methods Female nurses $(n=119)$ working irregular rotating shifts were recruited from two hospitals in Northern Israel, using convenience sampling by clusters. Inclusion criteria were working at least $75 \%$ of full time, with at least one night shift per week. Exclusion criteria were pregnancy, a diagnosed sleep disorder, and/or chronic medical conditions. Subjective sleepiness was measured hourly during two night shifts using the Karolinska Sleepiness Scale (KSS). Sleep was monitored by actigraphy 24 hours before and until the end of the night shifts. Participants completed a socio-demographic questionnaire, the Munich ChronoType Questionnaire for Shiftwork (MCTQ $^{\text {shift) }}$, the Pittsburg Sleep Quality Index (PSQI) and the Pre-Sleep Arousal Scale (PSAS).

Results Mixed models stepwise analyses found main effects for hour, age, cognitive pre-sleep arousal and number of children on nighttime sleepiness (all $\mathrm{p}<0.01$ ). Effects of chronotype on sleepiness were inconsistent. Interactions were found for age *number of children $(\mathrm{p}<0.01)$, pre-sleep cognitive arousal*chronotype $(\mathrm{p}<0.05)$, and age* chronotype $(\mathrm{p}=0.06)$. Older nurses were less sleepy than younger nurses, but this impact was attenuated by early chronotype and having more children. High cognitive pre-sleep arousal, but not sleep, predicted increased sleepiness, especially in nurses with late chronotype. Discussion The impact of bio-psycho-social factors on night shift sleepiness is complex and depends on mutual interactions between these factors. Nurses who are young, late chronotypes and with high cognitive pre-sleep arousal require special attention and support, and must develop personal strategies for maintaining vigilance on the night shift.

\section{2d BREAST CANCER AND SHIFT WORKING IN A LIGHT POLLUTED WORLD}

Abraham Haim*, Zubidat Abed. The Israeli Centre for Interdisciplinary Research in Chronobiology, University of Haifa, Haifa, Israel

\subsection{6/oemed-2018-ICOHabstracts.1384}

Introduction Breast cancer (BC) is increasing worldwide together with light pollution (LP) emerging from various outdoor and indoor sources. Results of different studies including our research centre report on the relations between BC-incidences and exposure to Artificial Light at Night (ALAN). The trend for energy saving-ALAN increases the problem, as light intensity is increasing and mainly that of short wavelength (SWL), within the blue part of the spectrum $(450-500 \mathrm{~nm})$. Our master biological clock, located in the hypothalamus, entrained by light/dark cycles is in charge of our temporal organisation from cell functions. It is not only light-intensity, which changes with the 24 hour cycles, but also the dominant parts of the light spectrum, which reach's earth. Those dominant parts, signalling for daytime are the SWL, including those between $450-500 \mathrm{~nm}$ a range known as an efficient suppressor of the nocturnal pineal produced hormone Melatonin (MLT). We attempted to study the nexus: ALAN, MLT-
Suppression, epigenetic modifications and BC-cells proliferation in subcutaneously inoculated female mice.

Methods Mice were acclimated for two weeks under 8L:16D, at a constant ambient temperature testing various sources of illumination differing in spectrum composition. After inoculation, we exposed mice to ALAN of the same illumination of daytime. We measured the following variables: Body mass, tumour volume, MLT-production and levels of Global DNA methylation (GDM) levels.

Results We revealed the existence of the proposed nexus. Response to ALAN is depended on the wavelength illumination source. SWL-illumination bulbs as white-LED or compact florescent have a higher negative effect compared with that of incandescent or carbon bulbs. We emphasise a relation between tumour volume, level of MLT-suppression and GDMlevels.

Discussion We suggest that human populations under increasing LP-levels of SWL-illumination are in a high risk for becoming BC-patients, it should be of great interest to set the threshold for exposure to SWL-illumination and BC-risk.

\section{2e CAN ON-SHIFT NAP BENEFIT NIGHT WORKERS' HEALTH? STUDIES ON BLOOD PRESSURE AND OBESITY IN NURSING TEAMS}

${ }^{1}$ Lucia Rotenberg*, ${ }^{2}$ Aline Silva-Costa, ${ }^{1}$ Rosane $\mathrm{H}$ Griep. 'Laboratory of Health and Environmental Education, Oswaldo Cruz Foundation, Rio de Janeiro, Brazil, ${ }^{2}$ Department of Collective Health, Federal University of Triângulo Mineiro, Uberaba, Minas Gerais, Brazil

\subsection{6/oemed-2018-ICOHabstracts. 1385}

Introduction On-shift napping can benefit night workers as regards sleep deprivation, adjustment of circadian rhythms, and alertness. But possible benefits of nap on health outcomes are scarcely investigated. Considering that night work is pointed as a risk factor for hypertension and obesity, we investigated the possible attenuation of blood pressure and body mass index increase by on-shift napping among nursing teams.

Methods A cross-sectional study was conducted in a public hospital in Rio de Janeiro, Brazil with nursing professionals who were informally allowed to nap for up to three consecutive hours during working nights. Current and accumulated doses of night work (NW) were studied through the number of working nights/2 week-span and years of $\mathrm{NW}$, respectively. Four outcomes were measured using standard equipment and techniques: systolic blood pressure (SBP), diastolic blood pressure (DBP), hypertension (SBP $\geq 140 \mathrm{mmHg}$ or DBP $\geq 90$ $\mathrm{mmHg}$ or prescription of antihypertensive medication), and body mass index (BMI). The associations between exposure variables and outcomes were based on logistic regressions (hypertension) and generalised linear models (SBP, DBP and BMI).

Results Among non-nappers (but not among nappers), current doses of NW (number of working nights) was significantly associated with increased SBP ( $\beta$-value $=1.39 ; 95 \% \mathrm{CI}$ : 0.31 to 2.49 ) and DBP ( $\beta$-value $=0.80 ; 95 \% \mathrm{CI}$ : 0.10 to 1.50$)$, as well as increased odds for hypertension $(\mathrm{OR}=3.35$; 95\% CI: 1.74 to 6.57). This association was not observed for accumulated doses of NW. As regards BMI, both current and accumulated doses of NW were significantly associated with increased BMI levels $(\beta$-value $=0.364 \quad[95 \%$ CI: 0.002 to 0.749$]$ and $\beta$ value $=0.092$ [95\% CI: 0.011 to 0.173$]$, respectively) only among non-nappers. 
Discussion Despite difficulties to the practice of napping on the job, the promising results for both BP and BMI justify further investigations that could subsidise policies related to promoting adequate conditions for night workers to nap while on the job.

\section{$1602 f$ PRACTICAL GUIDELINES FOR LINKING BETTER WORKING TIME ARRANGEMENTS WITH STRESS PREVENTION AT WORK}

Kazutaka Kogi* ${ }^{\star}$ Y Sano. Ohara Memorial Institute for Science of Labour, Japan

\subsection{6/oemed-2018-ICOHabstracts. 1386}

Introduction Multifaceted work redesign is always involved in improving working time arrangements. Recent experiences in improving work systems with excessive work hours or overworking situations clearly show the need to link better working schedules with comprehensive measures to reduce stress at work. It is useful to know practical ways to facilitate joint changes or work schedules and job content.

Methods Typical types of improvements undertaken in participatory programmes for joint improvement of working time arrangements and job content for preventing stress at work were compared. The programmes studied included participatory occupational health activities of health care workers, local government employees and small enterprise workers. The common features of the participatory steps that facilitated the joint change process and the roles of trained facilitators were examined. The results were discussed to comile practical guidelines for linking better work schedules with other multifaceted stress-reducing improvements.

Results Multiple aspects addressed by the reviewed programmes commonly included team-based communication, work schedules, ergonomic work methods, physical environment and social support. Work schedule changes were usually combined with enhanced communication or improved work methods. It was found useful to utilise action-oriented tools such as action checklists reflecting local good practices and group work methods for proposing feasible improvements. New guidelines for organising participatory steps for the joint change of work schedules and job content were compiled with emphasis on simple group work procedures and the use of action-oriented checklists for proposing multifaceted actions.

Conclusions The participatory steps utilising action-oriented checklists and local good practices proved useful for facilitating planning and implementation of multifaceted improvements in work schedules and job content in the local context. It is suggested to organise participatory activities referring to the new guidelines compiling these positive features in linking working time arrangements and stress prevention at work.

\section{$1602 \mathrm{~g}$ SHIFTWORK, AUTOMATED VEHICLES, AND FUNCTIONAL IMPAIRMENTS IN TRANSPORTATION}

D Fisher*, S Popkin, M Lohrenz, K Davies-Schrils, D Moore. U.S. Department of Transportation, Volpe Centre, Cambridge, USA

\subsection{6/oemed-2018-ICOHabstracts. 1387}

Introduction Shiftwork has been linked to functional impairments such as fatigue and distraction that increase crash risk. Automated vehicles may decrease these risk factors. Simultaneously, automated vehicles will allow a broader range of 'drivers' to operate the vehicle such as those with cognitive and physical impairments, creating additional benefits and impacts to shiftworking drivers with such impairments. We have developed a conceptual model of how automation at all levels can be used to reduce the impacts of shiftwork on functional impairments in transportation.

Methods Over 100 articles were reviewed in the areas of shiftwork, automation, and functional impairment using established key words and recognised search domains. Central to the understanding of the relations among shiftwork, automation and functional impairments is knowledge of how drivers use, misuse, disuse and are abused by automation.

Results The result of the literature search and its analysis is a conceptual model which clarifies how shiftwork impacts the use, misuse and disuse of automation at each level, and how those impacts affect drivers with different types and levels of functional impairment. Existing research suggests that long shifts can lead to an increase in the misuse of automation, with fatigued and distracted drivers over-trusting automation. This effect will be magnified for those with cognitive impairments. However, systems are now deployed that can detect driver state such as fatigue and distraction, and could potentially communicate with an automatic driving suite and intervene when the driver is fatigued or distracted.

Discussion The conceptual model creates a roadmap for future research, applications and regulations that various stakeholders can use to improve the safety and well-being of shiftworkers.

\section{SHIFT WORK AS OCCUPATIONAL STRESS RISK FACTOR}

I Bukhtiyarov*, M Rubtsov. FSBSI 'Izmerov Research Institute of Occupational Health', Moscow, Russia

10.1136/oemed-2018-ICOHabstracts. 1388

The analyses of international and domestic publications shows that shift work is different professional groups occupational stress risk factor. From 2010 according IARC conclusion shift work is probable occupational cancer risk factor ('2A') primarily, breast cancer in women (nurses). The probability of association between shift work and increased of any other cancer and another occupations as well as connexion with frequency and duration of night shift work is discussed today.

There are bidirectional relationship between sleep disorders and work connected with psychosocial risks (style of work, high level of requirements and low level of control, working hours and shift mode, as well as imbalance between labour costs and remuneration Including). Desynchronosis in the shift mode, especially with rotation shifts, has negative stressful effect on physical and mental health, leading to increased risk of insomnia, restless leg syndrome, shift work sleep disorder, narcolepsy, cataplexy, obstructive sleep apnea syndrome, cardio-vascular disorders, metabolic syndrome, diabetes, and disorders of nervous system state and mental health.

The current hygienic classification in Russia makes it possible to assess the contribution of labour intensity as stress factor, including taking into account shifts. Analysis of shift work impact into risk of health disorders of law enforcement officers, doctors, nurses, railway workers data shows the dependence of its development probability on the of work experience with the rotation shifts, age and sex, primarily in the part of causal relationships between the work schedule and hypertension in conjunction with metabolic disorders development. 